ge und vor dem Diebstahl von persönlichen Daten, und muss daher weiter wirkungsvoll verfügbar sein.

\section{Giesecke \& Devrient und Bundesdruckerei bündeln ihr Auslandsgeschäft für Regierungslösungen}

Giesecke \& Devrient (G\&D) und die Bundesdruckerei GmbH haben am 22. Mai 2014 den Vertrag zur Gründung eines gemeinsamen Joint Ventures unterzeichnet. In dem neuen Gemeinschaftsunternehmen mit dem Namen Veridos $\mathrm{GmbH}$ bündeln die Partner ihr internationales Geschäft mit Lösungen zur sicheren Identifikation wie beispielsweise Pass- und Ausweissysteme für Regierungen. Sitz des neuen Unternehmens ist Berlin. Ihr Deutschlandgeschäft, das Geschäft im privatwirtschaftlichen Markt sowie den Banknotendruck verantworten Bundesdruckerei und Giesecke \& Devrient weiterhin in Eigenregie.

Giesecke \& Devrient wird an Veridos einen Anteil von 60 Prozent halten, die Bundesdruckerei hält 40 Prozent der Firmenanteile. Veridos wird neben dem Sitz in Berlin eine Betriebsstätte in München, einen Produktionsstandort in Griechenland und weitere Tochtergesellschaften in Kanada, Mexiko und Brasilien haben und selbstständig auf dem internationalen Markt tätig sein. Die Aufsichtsgremien beider Unternehmen, die Gesellschafter von G\&D sowie das Bundesfinanzministerium als Eigner der Bundesdruckerei, haben der Gründung des Joint Ventures zugestimmt. Vorbehaltlich der kartellrechtlichen Freigabe wird Veridos ab Mitte 2014 den Geschäftsbetrieb aufnehmen.

„Die beiden renommierten deutschen Spezialisten für sichere Regierungslösungen ergänzen sich in ihrem Produktangebot und weiteren wesentlichen Bereichen optimal. Gemeinsam schaffen wir einen starken deutschen Systemanbieter in einem international stetig wachsenden Markt", so Dr. Walter Schlebusch, Vorsitzender der Geschäftsführung von Giesecke \& Devrient. „Das neue Unternehmen wird ein technologisch führender Komplettanbieter von ID-Lösungen und somit zuverlässiger Ansprechpartner für Regierungen weltweit." Ulrich Hamann, Vorsitzender der Geschäftsführung der Bundesdruckerei GmbH: „Weltweit steigt die Nachfrage nach Lösungen und Produkten rund um sichere Identitäten für den hoheitlichen Markt. Veridos wird von der Innovationskraft und langjährigen Expertise der Bundesdruckerei und G\&D in der Hochsicherheitstechnologie profitieren und ist somit optimal für den internationalen Wettbewerb aufgestellt."

Auf Projektbasis arbeiten Giesecke \& Devrient und die Bundesdruckerei seit einigen Jahren erfolgreich im internationalen Markt zusammen. Gemeinsam produzieren sie zum Beispiel den Führerschein für Lettland und haben im vergangenen Jahr einen Tender für die Ausgabe der elektronischen ID-Karte im Kosovo gewonnen. Gleichzeitig steigt die weltweite Nachfrage nach ID-Lösungen für den hoheitlichen Markt: So wächst etwa die Zahl der Flugreisenden jährlich um rund fünf Prozent. Beide Unternehmen liefern dafür wichtige Sicherheitslösungen. Zudem wird der Schutz von Identitäten immer wichtiger. Die Bedeutung von fälschungssicheren Ausweisdokumenten und Grenzkontrollsystemen, die eine zügige Abwicklung gewährleisten und gleichzeitig höchste Sicherheit garantieren, nimmt deutlich zu.

Beide Partner bringen technologisches Know-how, etablierte internationale Kundenbeziehungen und den reichhaltigen Erfah- rungsschatz ihrer Mitarbeiter in das Unternehmen ein. Das Portfolio reicht von Komplettlösungen für die Herstellung und Personalisierung von Passdokumenten, ID-Karten, Karten für das Gesundheitswesen und Führerscheinen über Ausgabe-, Personalisierungsund Dokumentenverwaltungssysteme bis hin zu Lösungen für die Grenzkontrolle sowie Personen-Identifizierung. Ein weiterer wichtiger Bestandteil des Angebots werden elD-Systeme zur Authentisierung und Absicherung elektronischer Transaktionen im Internet und zum Schutz der Kommunikation von Behörden sein. Insgesamt wird Veridos rund 400 Mitarbeiter beschäftigen.

\section{Trusted Services für neue Chipkarten im Gesundheitswesen}

Die Bundesdruckerei GmbH hat eine Ausschreibung der gematik Gesellschaft für Telematikanwendungen der Gesundheitskarte $\mathrm{mbH}$ gewonnen. Das Unternehmen stellt in den kommenden Jahren seine Lösungen und Services für die Public-Key-Infrastruktur (PKI) der zweiten Generation von Chipkarten im Gesundheitswesen für die Erprobungsphase in fünf Bundesländern bereit. Im Rahmen des Projekts sorgt die Bundesdruckerei für die Personalisierung und Ausgabe der Heilberufsausweise (HBA) für Ärzte, Psychotherapeuten und Zahnärzte sowie der Institutionskarten (SMC-B) für Einrichtungen im Gesundheitswesen (z. B. Krankenhäuser, Arztpraxen). Zudem stellt sie ein Online-Portal bereit, das die Berufsorganisationen beim Beantragungs- und Freigabeprozess der Ausweise technisch unterstützt.

Die Basis für ein innovatives Identitätsmanagement im Gesundheitswesen sind sichere Identitäten und Daten. Um die Telematikinfrastruktur im Gesundheitswesen langfristig zu schützen, ist es zudem notwendig geeignete Kryptoverfahren einzusetzen. Dies zu gewährleisten, ist eine komplexe Aufgabe, die die Bundesdruckerei unter dem BegriffFull ID |Management zusammenfasst. Deswegen wurde die Bundesdruckerei von der gematik $\mathrm{GmbH}$ damit beauftragt, Chipkarten der nächsten Generation ("G2") im Gesundheitswesen bis 2018 zu personalisieren, auszugeben und mit Supportund Schulungsleistungen zu unterstützen. Neben der Personalisierung der Heilberufsausweise (HBA) mit individuellen optischen Daten wie Lichtbild und Namen umfasst der Auftrag die Ausstattung der HBAs mit Zertifikaten. Die ausgegebenen Ausweise verfügen zudem über eine Qualifizierte Elektronische Signatur (QES), mit der ein Arzt rechtssicher digital unterschreiben kann. Die verwendeten Zertifikate werden von D-TRUST, dem akkreditierten Trustcenter der Bundesdruckerei, in Berlin erzeugt, das auch die dafür notwendigen PKI-Lösungen bereitstellt.

Im Rahmen des Projekts entwickelt die Bundesdruckerei ebenfalls ein benutzerfreundliches Online-Portal, über das Ärzte, Zahnärzte, Psychotherapeuten und Krankenhäuser ihren Ausweis beantragen, und die zuständigen Berufsorganisationen die Prüfung und Freigabe durchführen können. Das Projekt ist Bestandteil des Gesamtvorhabens der gematik für die Einführung, den Betrieb und die Weiterentwicklung der Telematikinfrastruktur im Gesundheitswesen, der elektronischen Gesundheitskarte (eGK) sowie zugehöriger Fachanwendungen für die Datenkommunikation zwischen Heilberuflern, Kostenträgern und Versicherten. 